\title{
Scientific Literature of Small-Angle X-Ray and Neutron Scattering Applied to the Characterization of Materials at the Nanometer Scale
}

\author{
Hannes Fischer ${ }^{a} * \mathbb{D}$, Aldo Craievich \\ ${ }^{a}$ FATEC Shunji Nishimura, 17580-000, Pompeia, SP, Brasil \\ ${ }^{b}$ Instituto de Física, Universidade de São Paulo, 05508-090 São Paulo, SP, Brasil
}

Received: May 1, 2019; Revised: July 18, 2019; Accepted: September 18, 2019

\begin{abstract}
The SAXS and SANS techniques are widely applied to the structural characterization of materials at the nanometer scale. We here report quantitative analyses of the published literature related to these techniques and to relevant features of the International SAS Conferences. A total number of 42,048 articles related to SAXS and SANS were retrieved. The annual total number of published articles related to SAXS exhibits a fast and persistent growth from 1990 until 2018 while those referring to SANS remained essentially constant along the past two decades. Furthermore, the number of citations of articles published by authors from the USA and from some European countries (Germany, France, UK) are significantly higher than those published by authors from other countries. The persistent increase in the number of published articles related to SAXS around the world and particularly in Brazil is well correlated with the also increasing number of open synchrotron sources.
\end{abstract}

Keywords: $S A X S$, SANS, bibliometric analysis, trends in science.

\section{Introduction}

Small-angle X-ray and neutron scattering techniques (SAXS and SANS, respectively) are widely used for structural characterizations of heterogeneous materials at the nanometer scale. The first particularly relevant application of the SAXS technique to materials science was published in 1938 by Andre Guinier ${ }^{1}$, who experimentally demonstrated the existence of coherent $\mathrm{Cu}$-rich nano-aggregates embedded in the matrix of Al-Cu alloys (GP zones) which explained the effect induced by aging on the mechanical hardening of these alloys. The SANS technique started to be applied 30 years later, at the beginning of the 1970 s, when neutron sources (nuclear reactors) became available for this type of experiments. At present more than 42,000 articles related to these techniques were published, most of them during the last three decades.

The basic theory of the SAXS technique is essentially the same as that of SANS and is described elsewere ${ }^{2,3}$. Several applications of SAXS and SANS were reported in references ${ }^{4}$ and $^{5}$, respectively. For structural studies of thin films deposited on flat substrates, SAXS and SANS measurements are conducted at glancing incidence angles; in these cases, the techniques are named GISAXS and GISANS, respectively. ASAXS is another related technique that uses the effect of anomalous or resonant small-angle scattering.
The basic theory and some applications of GISAXS and ASAXS are described in reference ${ }^{6}$.

The main purpose of this article is to present a quantitative analysis of the relevant features of the scientific literature published during the last three decades related to the smallangle scattering (SAS) techniques, including those referring to SAXS, GISAXS, ASAXS, SANS, and GISANS. For this purpose we have retrieved in Web-of-Science essentially all articles related to these fields published from 1991 until July 2018 and determined the time dependences associated to different countries and journals. Other purposes are to identify the articles that received the highest number of citations and the time dependence of the number of presentations at the triennial International SAS Conferences.

The data-mining process was performed in two successive searching steps using different key-word sets. For the first search step, the topics were ((saxs or (small-angle near/2 scatter*) or gi\$saxs or gi\$sas or gi\$sans or (small-angle near/2 solution scattering))). This first step yielded 40,174 articles. The topics for the second search step were "SANS" minus the topics retrieved with the first step. This additionally yielded 5906 articles, but many of them are false positives, i.e., those corresponding to SANS words not related to small angle scattering. Here we only have a small subset left and in order to get only articles related to the SANS technique, we filtered this second set of articles by only preserving those corresponding to the following 
Web-of-Science fields: physics nuclear or energy fuels or physics particles fields or multidisciplinary sciences or chemistry physical or chemistry multidisciplinary or polymer science. Indexes: sci-expanded, ssci, a\&hci, cpci-s, cpci-ssh, esci. After this procedure, the second search step yielded 1,874 articles. These records were added to those corresponding to the first search query, thus finally yielding a total number of articles $\mathrm{N}(\mathrm{SAS})=42,048$ articles.

We have also separately determined the number of articles $\mathrm{N}(\mathrm{SAXS})$ and $\mathrm{N}(\mathrm{SANS})$ related to investigations associated with small angle X-ray and neutron scattering, respectively. Notice that a minor fraction of articles $(<5 \%)$ does not explicitly contain search words that may allow us to safely assign them to SAXS or to SANS, such as those only containing the search words "solution scattering", "small angle scattering" or "SAXS and SANS". In our analysis, half of this minor fraction of articles were counted as N(SAXS) and half as $\mathrm{N}(\mathrm{SAXS})$ thus resulting $\mathrm{N}(\mathrm{SAS})=\mathrm{N}(\mathrm{SAXS})+\mathrm{N}(\mathrm{SANS})$.

We only analyzed here the results corresponding to the articles published from 1991. The reason for this is twofold: (i) the numbers of articles related to SAXS or/and SANS published before 1991 were very low, and (ii) for retrieving articles from Web-of-Science published before 1991, the searching topic words should be included in the title while for those published from 1991 until July 2018, the topic words should be in their titles, keywords or abstracts. Notice that more than one-half of articles related to smallangle scattering do not mention the words SAXS or SANS in their title and, therefore, many articles related to SAXS or SANS published before 1991 cannot be retrieved.

The total set of retrieved articles containing results related to SAXS and/or SANS was the database that we have used for further analyses. This database was used as input to the Vantage-Point software, version $11^{7}$, for different analyses of the published literature presented and discussed in the forthcoming sections.

The use of Vantage-Point software allowed for many types of analyses. We have focused our attention only on some relevant aspects of the published literature, namely (i) time evolution of the total number of published articles related to SAXS or/and SANS, (ii) time evolution of the numbers of articles published by authors from different countries, (iii) average number of citations of articles published by authors from different countries, (iv) trend of the number of articles published in different journals, (v) list of most cited articles and (vi) historical evolution of the number of articles authored by scientists from Latin-America.

We also recorded by direct search the numbers of lectures and communications presented at the successive International Conferences on Small-Angle Scattering, which are the main forum for discussions of new theoretical methods, instrumental developments and scientific applications of the SAXS and SANS techniques. Some relevant features referring to the historical trend of the number of presentations at these conferences will be reported.
Results and discussions included in this article were presented at the XVII International SAS Conference (Traverse City, October 2018) and at the XIV Annual Meeting of the Argentinian Society of Crystallography (Buenos Aires, October 2018), and follow other previously published analyses ${ }^{8,9}$.

\section{Historical Trends of the Number of Articles Related to SAXS or/and SANS}

The first analysis of the set of retrieved data consisting of 42,048 articles regards the time evolutions of the annual numbers of articles related to SAXS or/and SANS published in indexed journals from 1991 until July 2018. Considering that the search procedure was conducted during the first weeks of July 2018, we have estimated the total numbers of published articles in the current year (2018) as twice the retrieved values.

The annual numbers of articles related to SAXS, SANS and SAS (SAXS or/and SANS) published from 1991 until 2018 are plotted in Figure 1. We can notice that from 1991 until 2000 the annual numbers of published articles related to SAXS and those referring to SANS are similar and exhibit the same trends. However, the annual number of articles related to SAXS increased later at progressively increasing growth rate and reached 1,700/year circa in 2018, while the number of articles related to SANS remained essentially invariant at approximately 500/year.

The different trends observed in the time evolutions of the numbers of articles referring to SAXS and to SANS displayed in Figure 1 are probably caused by different concurrent factors, one of them being related to the number of X-ray photon and neutron sources available to users. The fast increase in the number of articles related to SAXS is correlated with the also increasing number and quality of new synchrotron sources that started their operation during the last two decades. On the other hand, the approximate invariance in the number of SANS articles during the last two decades (Figure 1) may be to some extent related to the nearly constant number of neutrons sources in operation, the shut-down of several neutron sources in Europe and the USA being balanced by the start-up of new neutron sources in Asia and Oceania.

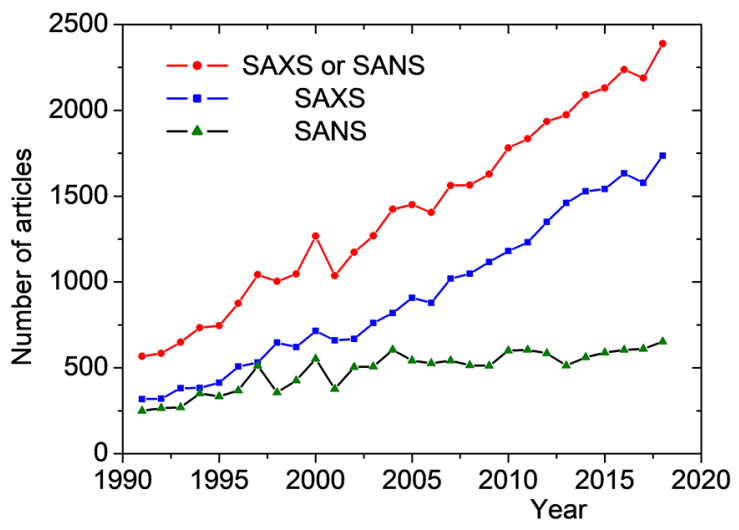

Figure 1. Annual numbers of published articles related to SAXS, SANS and SAS (SAXS or SANS). 
Moreover, a recent study ${ }^{10}$ also reported a nearly invariant number of articles, from 2005 to 2015 , related to the use of many neutron-based scattering techniques including SANS.

The constancy in the number of articles referring to SANS published during the last two decades may also be related to the high cost of neutron beam lines, which usually are more expensive than SAXS beam lines. In addition, high periods of time required for each sample analysis, complexity of the experiment, large amount of sample needed and management of radioactive samples (activated upon neutron irradiation) may also contribute to slow down the number of researchers choosing SANS as a primary technique. Notice that the number of users of the SAXS technique is much higher than that of SANS users even in Europe, where several reactor and spallation sources are available.

\section{Geographical Distribution of the Number of Articles and Average Numbers of Citations}

\subsection{Numbers of articles related to SAS (SAXS or SANS)}

The next step of this work was to establish the geographical distribution of the scientific literature related to SAS (SAXS or SANS) and their numbers of citations. In Table 1 we report the total number of articles published until July 2018 by authors from 41 countries that published at least a total number of 100 articles related to SAS. In this Table we have assigned the articles to the countries where their first authors are working. This procedure makes the article-country association easier and produces negligible bias effects.

As shown in Table 1, the total number of articles related to SAS (SAXS or SANS) published by authors from the USA is, by far, the highest $(11,280)$ followed by those from Germany $(6,948)$ and France $(6,428)$. Considering only the articles related to SAXS the most productive countries are also the USA $(8,550)$, Germany $(4,010)$ and France 3,475$)$. In the case of articles related to SANS, authors from France published the highest number $(2,953)$ followed by those from Germany $(2,938)$ and the USA $(2,730)$. Authors from fourteen countries published more than 1,000 articles related to SAXS or SANS, eight are from Europe (Germany, France, UK, Russia, Italy, Switzerland, Austria, and Netherlands), three from Asia (Japan, China, and India), two from the Americas (the USA and Brazil) and one from Oceania (Australia). Regarding the geographical distribution among the 41 countries with a total number of articles higher than 100 , they are from Europe (24), Asia (9), the Americas (5), Oceania (2) and Africa (1). Authors from most Latin-American, Asian and African countries published a lower number of articles $(\mathrm{N}<100)$.
The numbers reported in Table 1 reflect a heterogeneous geographical distribution of research activities related to SAXS or SANS, which are mainly concentrated in the USA and several European countries. Most of the countries with very low or inexistent scientific production are in Africa, some of them in Latin-America (all countries except Brazil, Mexico, and Argentina) and others in Asia. Another analysis not shown here indicates a clear correlation between the gross product per capita of different countries and their number of published articles related to SAS. Obviously, this basic correlation is a priori expected and probably applies to many other scientific fields.

Another interesting feature regarding the 20 countries with the highest numbers of published articles related to SAXS (Table 1) is that 17 of them have at least one synchrotron radiation source in operation, and three (Netherland, Denmark, and Poland) have access to synchrotron sources in neighbor countries. This reflects a relevant role of the use of synchrotron radiation sources for enhancing the production of SAXS articles. As will be shown in section 4, almost all SAXS articles published by authors from Latin-American countries contain experimental results from synchrotron SAXS beam lines.

Moreover, we have also compared the geographical distribution of the total numbers of articles related to SAS (either to SAXS or SANS) with the average numbers of citations per article corresponding to twelve countries with the highest total numbers. As shown in Figure 2, there is no clear correlation between the total numbers of articles published by authors from different countries and their average numbers of citations per article. Articles published by scientists from the USA, Germany, France, UK and Switzerland exhibit highest average numbers of citations, namely 30, 24, 27 and 24, respectively, while those published by authors from Japan, China, Russia, Italy, India, Australia, and Brazil received lower average numbers (below 20 citations/article).

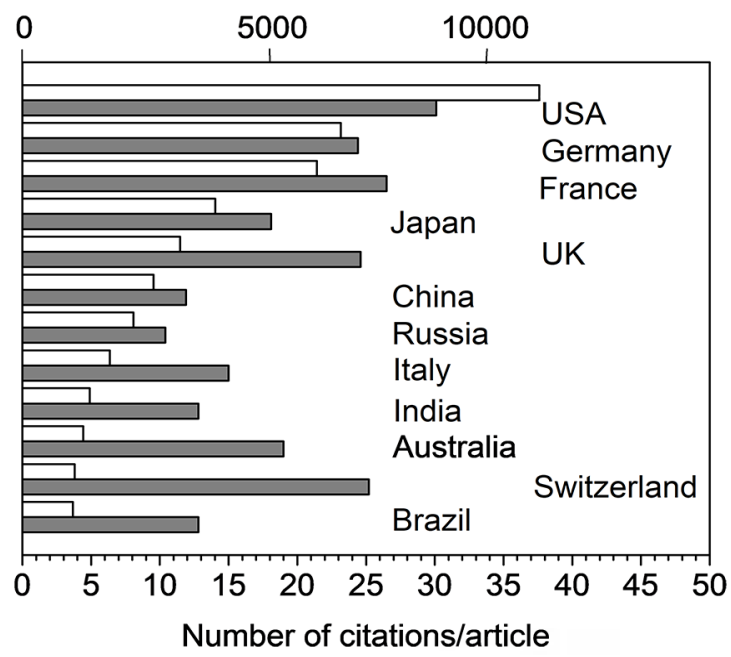

Figure 2. Empty bars: Total numbers of published articles related to SAXS or SANS (upper scale). Solid bars: Average citation numbers per article corresponding to countries with a total number of published articles $\mathrm{N}>1,000$. 
Table 1. Total numbers of articles published by authors from different countries related to SAXS, SANS and SAS (either SAXS or SANS).

\begin{tabular}{|c|c|c|c|c|}
\hline \# & Country & Number SAXS & Number SANS & Number SAS (SAXS or SANS) \\
\hline 1 & USA & 8550 & 2730 & 11280 \\
\hline 2 & Germany & 4010 & 2938 & 6948 \\
\hline 3 & France & 3475 & 2953 & 6428 \\
\hline 4 & Japan & 3428 & 783 & 4211 \\
\hline 5 & UK & 2449 & 990 & 3439 \\
\hline 6 & China & 2185 & 674 & 2859 \\
\hline 7 & Russia & 1598 & 825 & 2423 \\
\hline 8 & Italy & 1260 & 646 & 1906 \\
\hline 9 & India & 1260 & 211 & 1471 \\
\hline 10 & Australia & 886 & 443 & 1329 \\
\hline 11 & Switzerland & 873 & 265 & 1138 \\
\hline 12 & Brazil & 744 & 361 & 1105 \\
\hline 13 & Austria & 567 & 508 & 1075 \\
\hline 14 & Netherlands & 534 & 485 & 1019 \\
\hline 15 & South Korea & 527 & 437 & 964 \\
\hline 16 & Spain & 516 & 432 & 948 \\
\hline 17 & Sweden & 483 & 458 & 941 \\
\hline 18 & Denmark & 476 & 411 & 887 \\
\hline 19 & Canada & 449 & 388 & 837 \\
\hline 20 & Poland & 426 & 222 & 648 \\
\hline 21 & Belgium & 417 & 220 & 637 \\
\hline 22 & Taiwan & 317 & 316 & 633 \\
\hline 23 & Hungary & 308 & 254 & 562 \\
\hline 24 & Czech Rep. & 296 & 235 & 531 \\
\hline 25 & Israel & 274 & 189 & 463 \\
\hline 26 & Ukraine & 184 & 186 & 370 \\
\hline 27 & Greece & 184 & 169 & 353 \\
\hline 28 & Finland & 179 & 159 & 338 \\
\hline 29 & Portugal & 161 & 115 & 276 \\
\hline 30 & Argentina & 155 & 61 & 216 \\
\hline 31 & Norway & 131 & 81 & 212 \\
\hline 32 & Romania & 125 & 71 & 196 \\
\hline 33 & Singapore & 125 & 51 & 176 \\
\hline 34 & Slovakia & 114 & 55 & 169 \\
\hline 35 & Croatia & 112 & 47 & 159 \\
\hline 36 & Iran & 96 & 63 & 159 \\
\hline 37 & Mexico & 92 & 61 & 153 \\
\hline 38 & Slovenia & 85 & 57 & 142 \\
\hline 39 & N. Zealand & 71 & 68 & 139 \\
\hline 40 & Turkey & 65 & 48 & 113 \\
\hline 41 & S. Africa & 59 & 45 & 104 \\
\hline
\end{tabular}

The clear differences observed in the average numbers of citations of articles from geographically central countries such as the USA, Germany, France and UK and those from peripheric countries, namely Japan, China, Russia, India,
Australia and Brazil, may be assigned to a different average quality of the articles published by central and peripheric countries and/or to some bias in the selection of the cited literature by authors from central countries. 
The observed differences in the number of citations of articles published by authors of central and peripheric countries may also be due to eventual differences in the impact factors of the journals in which articles are published. However, for clearly establishing the relevant reasons that explain the mentioned differences, additional and deeper analyses are required

\subsection{Historical trend of SAXS-related articles}

We have analyzed the time dependences of the annual numbers of articles related to SAXS published by authors from different countries from 1991 until July 2018. As in the previous section, we have also assigned each article to the country where the first author is working. This analysis refers only to the literature published by authors from a set of ten countries with highest publication rates in 2018 .

Figure $3 \mathrm{a}$ and $3 \mathrm{~b}$ exhibit the annual number of articles published by authors from countries with the highest numbers of published articles related to SAXS in 2018. Figure 3a displays the results corresponding to China, the USA, Germany, Brazil and Japan, which in 2018 published 308, 260, 168, 94 and 86 articles, respectively. We can notice that the number of articles corresponding to Japan remained approximately constant during the last decade, those corresponding to the USA, Germany and Brazil exhibit clearly growing trends and that referring to China shows a fast growth and an also increasing growth rate.

The results plotted in Figure $3 \mathrm{a}$ indicate that in 2018 the annual number of articles related to SAXS written by authors from China became similar to those written by American authors. Moreover, if both, the current fast growth in the number of articles by authors from China and the moderate growth rate in the number of articles written by authors from the USA persist, Chinese authors will soon publish the highest annual number of articles referring to SAXS.

Another conclusion derived from the results plotted in Figure $3 \mathrm{a}$ is that the annual number of articles published by Brazilian authors in 2018 became similar to the number of articles published by Japanese scientists.
As will be discussed in Section 4, the rather fast growths of the number of articles published by Brazilian and Argentinean authors are essentially related to the availability and intense use of the Brazilian synchrotron X-ray source that is in operation from 1997.

The results plotted in Figure $3 \mathrm{~b}$ indicate that the numbers of SAXS articles published by authors from UK, Italy and Australia exhibit some oscillation and reach a nearly constant value (from 40 to 60 articles) during the last decade. On the other hand, the number of SAXS articles published by authors from Russia maintains a growing trend up to about 65 articles per year in 2018, while those corresponding to France reached a maximum above 100 in 2013 and evidence a decreasing trend during the last few years, down to $\sim 80$ in 2018 .

\subsection{Historical trend of SANS related articles}

Figure 4a displays the annual number of articles related to SANS published by first authors from the five countries with highest production in 2018 (the USA, Germany, India, Russia and UK). During the last two decades, the annual numbers of articles from the USA and Germany exhibit oscillations but maintain an overall constant trend, while those from UK show a persistent constant value. On the other hand, the annual numbers of articles published by authors from India and Russia evidence clearly increasing trends.

In Figure $4 b$ we have plotted the annual numbers of published articles related to SANS corresponding to authors from other countries with lower annual numbers in 2018 (from $\sim 10$ to 40 articles), which show persistent growths for authors from China and Australia and clear decreasing trends for those from France, Japan, and Italy

Data plotted in Figures $4 \mathrm{a}$ and $4 \mathrm{~b}$ indicate that the number of articles published during the last decade by authors from India, Russia, China, and Australia evidence clear growths, those from the USA, Germany, and UK exhibit nearly constant values, and those from France, Japan and Italy show decreasing trends.
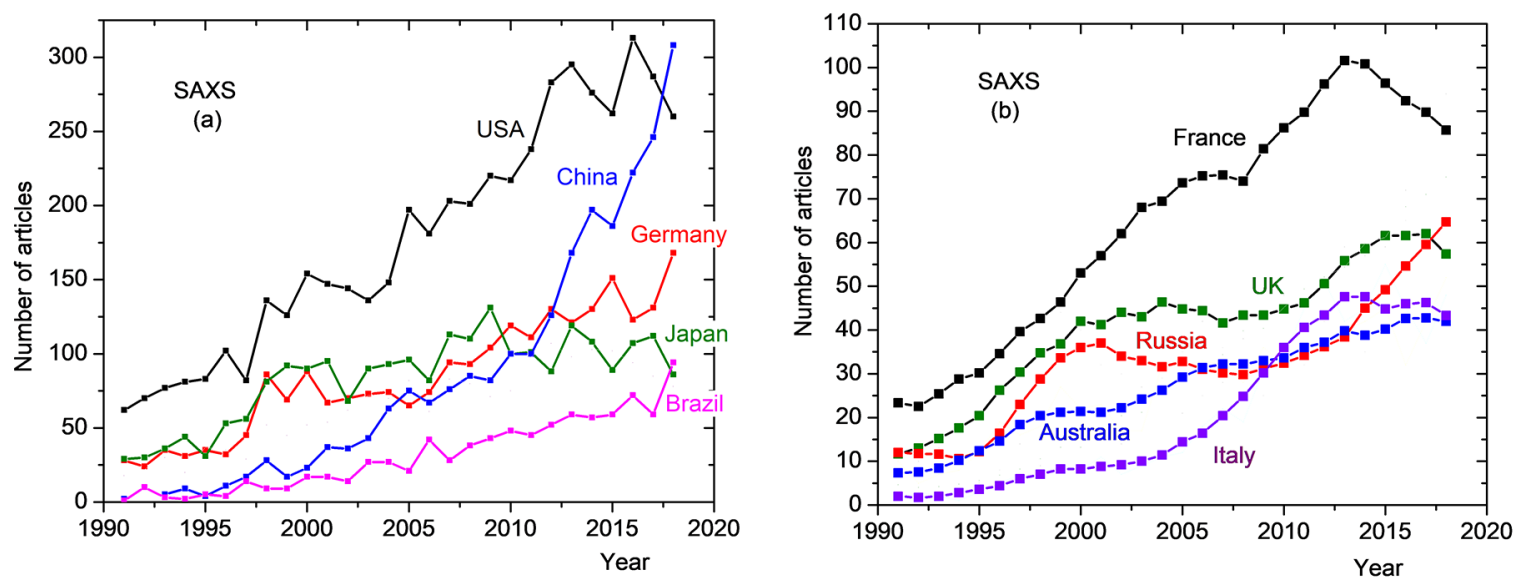

Figure 3. Historical evolution of the annual number of articles related to SAXS corresponding to the ten most productive countries in 2018 . (a) Countries for which the number of articles published in 2018 range from $\sim 80$ up to 310. (b) Countries for which the number of articles published in 2018 range from $\sim 50$ up to 80 . Data in (b) were smoothed by five adjacent point averaging. 
These different trends of the number of published articles by authors from the ten most productive countries are consistent with the already mentioned nearly invariant number of articles related to SANS published during the last two decades (Figure 1).

\section{Distribution Among Journals}

Another purpose of this work was to identify the journals in which the highest total number of articles related to SAS (either SAXS or SANS) were published. As shown in Figure 5a, the journals that published the highest numbers of articles related either to SAXS or SANS are, in decreasing order, Macromolecules, Polymer, Langmuir, Journal of Applied Crystallography, Journal of Polymer Science, Journal of Physical Chemistry, Journal of Biological Chemistry, Journal of Applied Polymer Science, Biophysical Journal and Journal of Colloids and Interfaces.
Notice that among the mentioned 10 journals only three (J. Appl. Cryst. J.Phys. Chem. and J. Colloids and Int.) publish articles dealing with either soft and hard matter while the other seven journals only publish articles reporting applications to soft matter. This implies that SAS techniques are predominantly applied to structural characterizations of soft matter and to a lesser extent to studies of hard (inorganic) matter. Most of the articles dealing with soft matter report structural investigations of polymers in solid state and roughly about $10 \%$ to biological systems such as proteins in solution.

The annual numbers of articles published in all journals listed in Figure 5a indicate a persistent increasing trend with different growth rates. Because of the fast growth of the total number of published articles reported in Figure 1, these increasing trends are a priori expected. Furthermore, we have determined the fractions of articles related to SAS with respect to the total number of articles published in each journal, which exhibit for most of them clearly increasing trends.
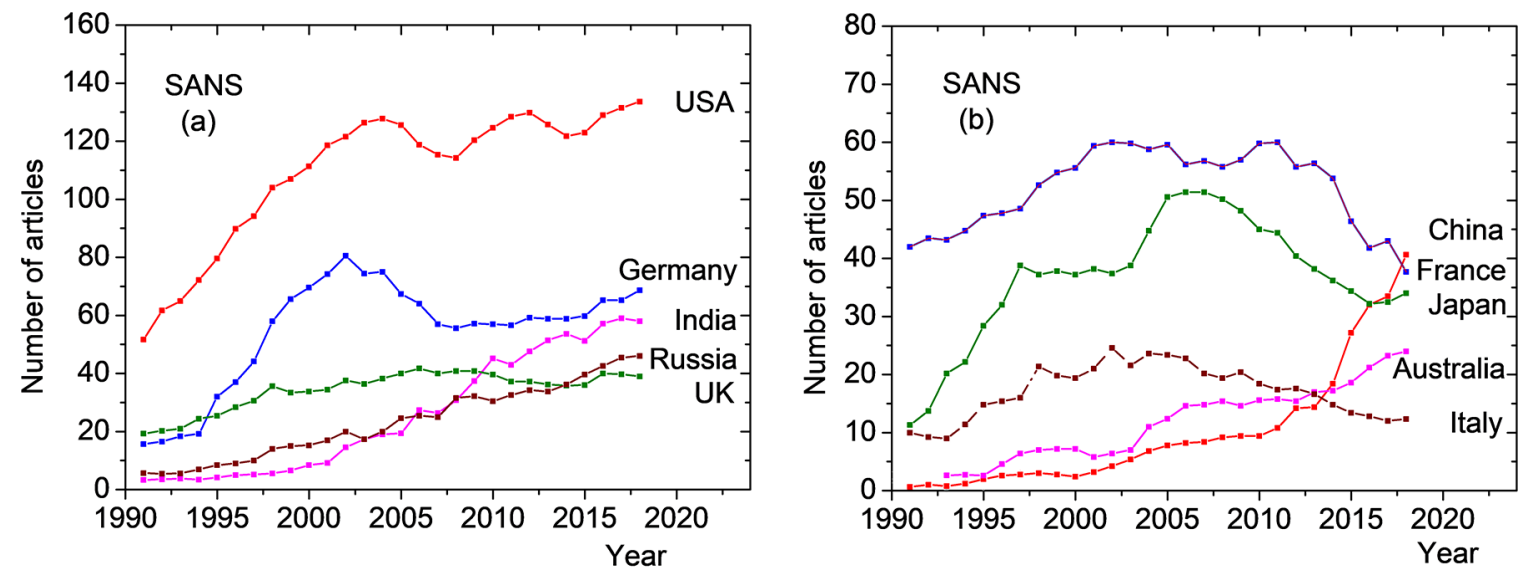

Figure 4. Historical evolution of the annual number of articles related to SANS published by authors from the ten most productive countries in 2018. (a) Countries for which the number of articles published in 2018 range from $\sim 50$ up to 150. (b) Countries for which the number of articles published in 2018 range from $\sim 10$ up to 40 . Data in (a) and (b) were smoothed by five adjacent points averaging.

\begin{tabular}{|c|c|}
\hline (a) & 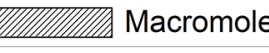 \\
\hline & Polymer \\
\hline 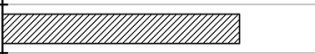 & Langmuir \\
\hline 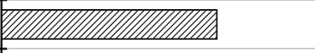 & J Appl Cryst \\
\hline 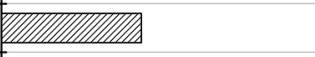 & J Polymer Sci \\
\hline प्रायागय & J Phys Chem \\
\hline 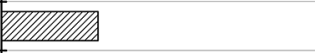 & J Biol Chem \\
\hline 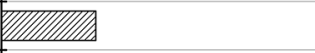 & J Appl Polymer Sci \\
\hline क्याओ & Biophysical J \\
\hline (1) & $\mathrm{J}$ Colloids and Int \\
\hline 1000 & 1500 \\
\hline
\end{tabular}

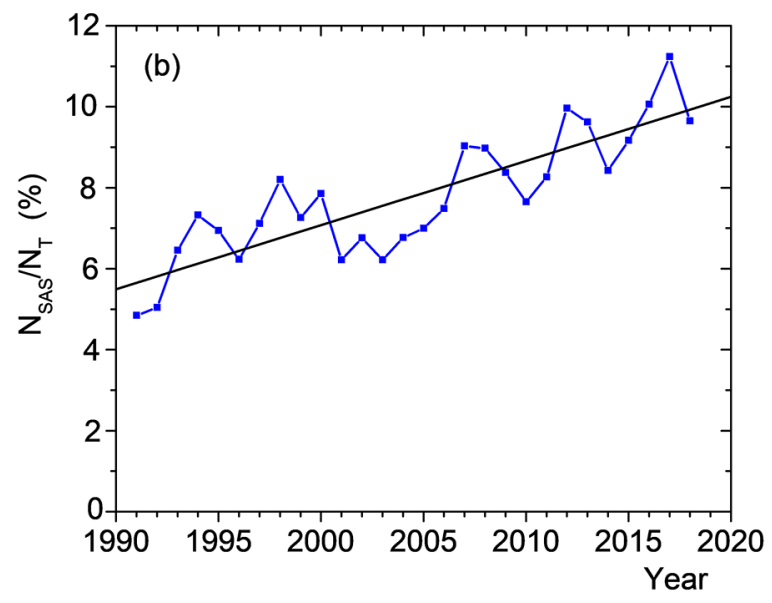

Figure 5. (a) Total number of articles related to SAXS or SANS published by different journals. (b) Historical evolution of the ratio of the number of articles related to SAXS or SANS with respect to the total number of articles published in Macromolecules. The straight line is a guide for the eye. 
This result evidences an increasing relative contribution from SAS-related research fields to the general scientific literature. Figure $5 \mathrm{~b}$ shows this effect for the particular case of the journal Macromolecules. Moreover, we report in Table 2 the ratios of the number of articles related to SAS - published in different journals - with respect to the total numbers of published articles, corresponding to 1991 and 2018. The quotients between both fractions also reported in Table 2 indicate a fast growth during the last three decades of the fraction of articles related to SAS with respect to the total number of published articles.

In order to identify the most cited articles related to SAXS or SANS, we have sorted the retrieved 42,048 articles by decreasing citation numbers. Table 3 reports details of 14 references with more than 1,000 citations. Notice that only two articles have received more than 1,000 citations until $2008^{9}$. Among 14 articles with the highest citation numbers, five were published in Journal of Applied Crystallography, three in Chemical Reviews, and the others in different journals. Three articles consist of reviews of different scientific fields and six refer to methods of data analysis. These highly cited articles were authored by scientists from the USA (5) and from different European countries, namely Germany (6), France (1). Russia (1) and Austria (1).

\section{Number of Published SAS Articles and Use of Synchrotron Radiation in Latin-America}

Most of the articles related to SAS published by authors from Latin-America are associated with SAXS experiments while only a minor fraction are derived from SANS results. The relatively low number of articles related to SANS is very probably due to the current absence of neutron sources with neutron beams in this region of the world. For this reason, we will here only discuss the aspects associated with the historical trend and geographical distribution of the number of articles related to SAXS.

The first articles related to SAXS authored by scientists from the USA and Europe were published in the decade of 1930 while the first article related to this type of investigation written by Latin-American authors was published 30 years later. The results of our data-mining indicates that the first investigation using SAXS in Latin-America was performed in Argentina and dealt with pre-precipitation in AlZn alloys ${ }^{25}$.

Table 2. Ratios of the number of articles related to SAS with respect to the total number of articles published in selected journals in 1991 and 2018. The third column reports the quotient between both ratios.

\begin{tabular}{lccc}
\hline Journal & $\mathrm{C}(1991)$ & $\mathrm{C}(2018)$ & $\mathrm{C}(2018) / \mathrm{C}(1991)$ \\
\hline Macromolecules & 5.5 & 10.0 & 1.81 \\
Langmuir & 3.0 & 7.0 & 2.33 \\
J. of Applied Crystallography & 6.0 & 17.0 & 2.83 \\
Polymer & 3.8 & 5.5 & 1.43 \\
\hline
\end{tabular}

Table 3. Articles related either to SAXS or SANS with more than 1,000 citations by July 2018 . The table reports citation numbers, titles of articles, references and countries of first authors.

\begin{tabular}{|c|c|c|}
\hline Citations & Title & Country \\
\hline 3,050 & $\begin{array}{l}\text { (CSe)ZnS core-shell quantum dots. Synthesis and characterization of a size series of highly luminescent } \\
\text { nanocrystallites. }{ }^{11}\end{array}$ & USA \\
\hline 2,466 & Low molecular mass gelators of organic liquids and the properties of their gels. ${ }^{12}$ & France \\
\hline 2,235 & $\begin{array}{l}\text { Buildup of ultrathin multilayer films by a self-assembly process consecutively alternating adsorption of } \\
\text { anionic and cationic polyelectrolytes on charged surfaces. }{ }^{13}\end{array}$ & Germany \\
\hline 2,145 & Determination of the regularization parameter in indirect-transform methods using perceptual criteria. ${ }^{14}$ & Germany \\
\hline 1,967 & CRYSOL. A program to evaluate X-ray solution scattering of biological macromolecules. ${ }^{15}$ & Germany \\
\hline 1,583 & PRIMUS. A Windows PC-based system for small-angle scattering data analysis. ${ }^{16}$ & Germany/Russia \\
\hline 1,545 & $\begin{array}{l}\text { Alkanethiolate gold cluster molecules with core diameters from } 1.5 \text { to } 5.2 \mathrm{~nm} \text { : Core and monolayer properties } \\
\text { as functions of core size. }{ }^{17}\end{array}$ & USA \\
\hline 1,459 & Water gelation by small organic molecules. ${ }^{18}$ & USA \\
\hline 1,262 & Connection between polymer molecular-weight, density, chain dimensions, and melt viscoelastic properties. ${ }^{19}$ & Germany \\
\hline 1,252 & $\begin{array}{l}\text { Restoring low-resolution structure of biological macromolecules from solution scattering using simulated } \\
\text { annealing. }{ }^{20}\end{array}$ & Germany \\
\hline 1,177 & Uniqueness of ab initio shape determination in small-angle scattering. ${ }^{21}$ & Germany \\
\hline 1,144 & Structure and process relationship of electrospun bioabsorbable nanofiber membranes. ${ }^{22}$ & USA \\
\hline 1,094 & Supramolecular nanotube architectures based on amphiphilic molecules. ${ }^{23}$ & USA \\
\hline 1,013 & New method for evaluation of small-angle scattering data. ${ }^{24}$ & Austria \\
\hline
\end{tabular}


Moreover, the first application to materials science using SAXS in Brazil published 8 years later was an experimental study of phase separation in a $\mathrm{B}_{2} \mathrm{O}_{3}-\mathrm{PbO}-\mathrm{B}_{2} \mathrm{O}_{3}$ glass $^{26}$.

Almost all articles related to SAXS corresponding to Latin-American countries were written by authors from Brazil, Argentina, and Mexico, which published until July 2018 a total number of 886,161 and 96, respectively. Figure 6 a displays the numerical evolution of the scientific literature related to SAXS published by authors from these countries. The numbers of articles published by authors from Brazil and Argentina started from almost zero by 1990 and from then exhibit a persistent increasing trend and an also increasing growth rate until 2018.

Another purpose of this investigation was to establish to what extent the use of the SAXS beam lines of the Brazilian National Synchrotron Light Laboratory (LNLS), which is in operation from 1997, has contributed to the development of SAXS-related fields of research in Latin-America. Taking into consideration that almost all users of the LNLS SAXS beam lines are Brazilian and Argentinian scientists, we have plotted in Figure $6 \mathrm{~b}$ the time dependence of the added annual numbers of articles published by authors from both countries that contain experimental results obtained by using the LNLS SAXS beam lines. These numbers were taken from public records corresponding to all LNLS beam lines [www.lnls.br]. On the other hand, we have also plotted in Figure $6 \mathrm{~b}$ the annual number of articles published by authors from Brazil and Argentina independently derived from our data-mining search in Web-of-Science. We can notice that both curves displayed in Figure $6 \mathrm{~b}$ overlap thus indicating that essentially all articles related to SAXS published by authors from Brazil and Argentina contain experimental results obtained by using the SAXS beam line of the Brazilian synchrotron laboratory. In other words, the results displayed in Figure $6 \mathrm{~b}$ implies that the persistent growths in the numbers of articles related to SAXS published by authors from Brazil and Argentina during the last two decades are due to the open availability of the SAXS beam lines associated with the locally available LNLS synchrotron source.

\section{Historical Trend of the Number of Presentations at International SAS Conferences}

We have also analyzed quantitative aspects related to the series of International Conferences on Small-Angle Scattering that are held every three years in different countries. The historical evolution of the number of lectures and communications presented in these Conferences was evaluated by counting the total numbers of abstracts included in the abstract booklets. These numerical results are displayed in Figure 7.

As shown in Figure 7, from the SAS Conference held at Hamburg in 1984 until that held at Kyoto in 2006, the number of scientific presentations exhibits a fast growth with a persistent positive variation in growth rate. This accelerated growth did not persist after the SAS Conference held in $2006^{9}$. As a matter of fact, the numbers of abstracts corresponding to the events held at Oxford in 2009, Sydney in 2012 and Berlin in 2015 indicate a starting trend toward an incipient plateau. Considering the numbers of presentations at different Conferences, from that held at Hamburg in 1984 until that at Berlin in 2015, and excepting the number at Kyoto in 2006, the time dependence is well described by a sigmoidal function, thus indicating a saturation effect starting in 2009, at 500 abstracts/conference circa. Moreover, a lower number of contributions (390) were presented at the Conference recently held at Traverse City, in October 2018. Because of differences in available logistic, funding ressources and/or physical space, the rather low number of presentations at the last International SAS Conference may be just an outlier number in plotted data thus not necessarily implying the beginning of a declining trend.
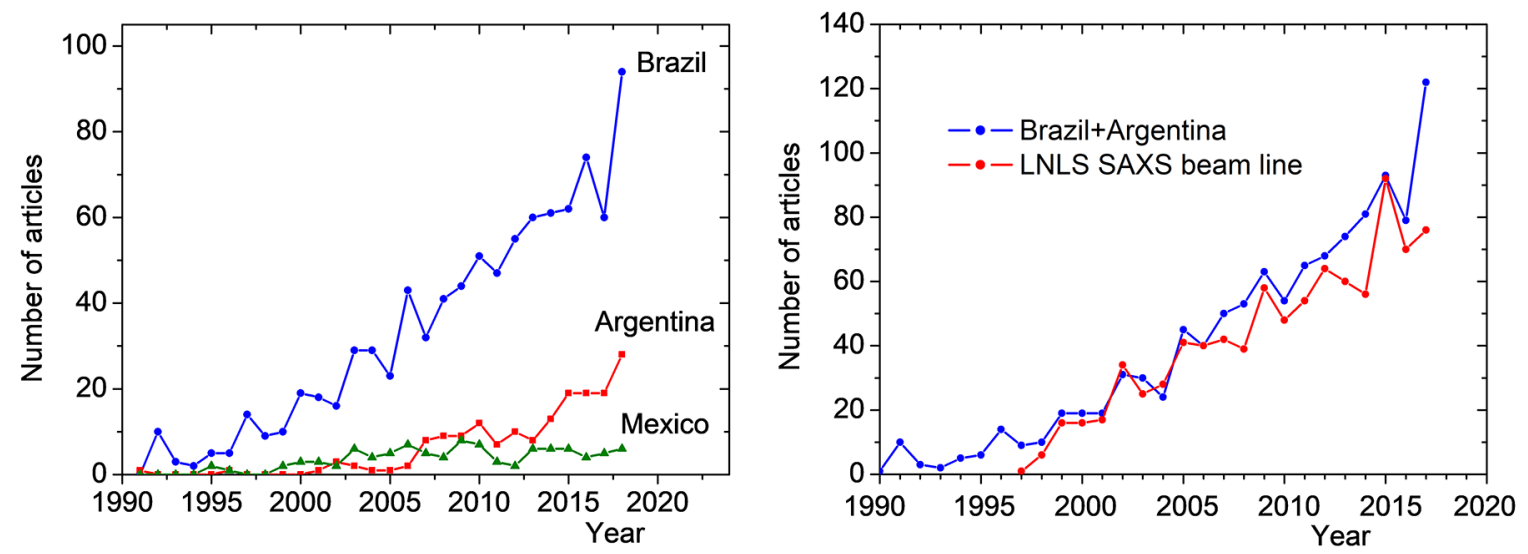

Figure 6. (right) Historical evolution of the annual numbers of articles related to SAXS published by authors from three Latin-American countries (Brazil, Argentina, and Mexico). The annual numbers of articles related to SAXS published by authors from other Latin-American countries are much lower, (left) Added annual numbers of articles related to SAXS published by authors from Brazil and Argentina. Blue symbols: Numbers determined by our Web-of-Science search procedure. Red symbols: Numbers determined by independent counting of published articles containing results from experiments using the LNLS SAXS beam lines. 


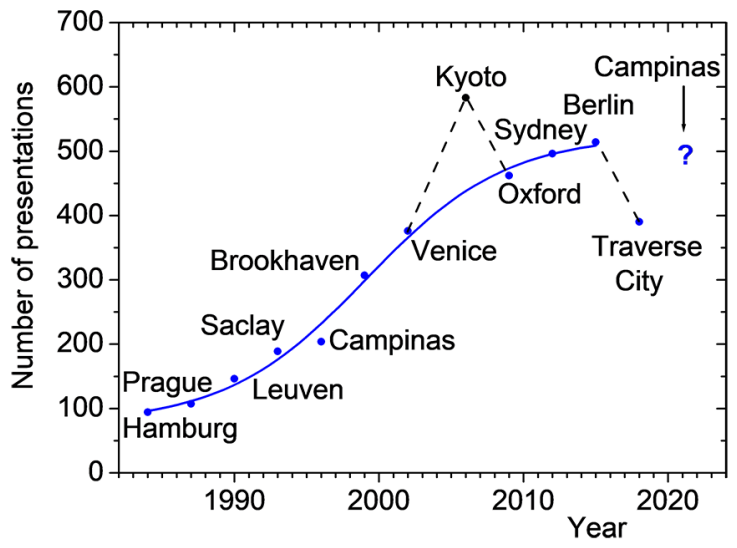

Figure 7. Number of abstracts referring to lectures and communications presented at the International SAS Conferences held at the different indicated places.

In conclusion, while the annual number of published articles related to SAS currently exhibits a strong and still persistent growth (Figure 1), the historical trend of the number of abstracts presented at the series of SAS Conferences seems to have reached an apparent upper limit of 500 abstracts circa that persisted over the last decade.

\section{Final Comments}

We have analyzed different numerical results referring to published articles related to SAXS or/and SANS, their historical trends, and their geographic distribution. These results led to the following main conclusions: (i) during the last three decades, the annual number of published articles related to SAXS exhibits a strong and persistent growth, this trend is correlated with the also increasing number and better quality of new synchrotron sources which started their operation during the last decades; (ii) along the past two decades, the annual number of published articles related to SANS remains essentially constant, which is probably related to the also nearly invariant number of neutrons sources in operation around the world; (iii) the historical evolution in the number of articles published by authors from different countries exhibit different (growing, constant or declining) trends; (iv) the annual number of articles published by authors from China exhibits a fast growth and reaches in 2018 a number similar to that from American authors; (v) the average numbers of citations of articles written by authors from the USA and some European countries (Germany, France, and the UK) are clearly higher than those written by authors from other countries; (vi) the time dependence of the fraction of articles related to SAS with respect to the total number of articles published in several journals is an increasing time-dependent function; (vii) the persistent growth in number of articles related to SAXS published by
South-American authors is mainly due to the open availability of local synchrotron SAXS beam lines, and (viii) only a relatively low number of articles related to SANS were up to now published by Latin-American authors, this situation is expected to be modified when the Argentinian Laboratory for Neutron Beams (LAHN) - now under construction in Buenos Aires - will be available to users.

On the other hand, the number of presentations at the series of International Conferences on Small-Angle Scattering exhibits a fast and persistent growth until 2005, a saturation effect at about 500 until 2015 and a lower value (390) in the last SAS Conference held in October 2018. Moreover, when the numbers of presentations at the forthcoming International SAS Conferences to be held in Campinas (Brazil) in 2021 and Taipei (Taiwan) in 2024 will be known, it will possible to verify if the number of presentations at Traverse City indicates the beginning of a declining trend or, otherwise, it is just an outlier point in the curve.

We expect that the results reported in this article will allow users to be aware of relevant features of the literature output from experiments using SAXS and SANS and motivate other materials scientists to utilize these techniques. This study may also assist decision makers dealing, for instance, with the forthcoming Brazilian synchrotron facility (Sirius), the project of the Brazilian Multi-purpose Reactor (RMB) and the on-going construction of the Argentinian Laboratory of Neutron Beams (LAHN).

\section{Acknowledgment}

The authors thank J. Trewhella, J. Teixeira and G. McIntyre for useful comments and acknowledge the financial support provided by the Brazilian National Science Council (CNPq).

\section{References}

1. Guinier A. Structure of age hardened Al-Cu alloy. Nature. 1938;142:569-570.

2. Guinier A, Fournet G. Small-Angle Scattering of X-rays. New York: John Wiley \& Sons; 1955.

3. Feigin LA, Svergun D. Structure Analysis by Small-Angle X-ray and Neutron Scattering. New York: Springer US; 1987.

4. Craievich AF. Small-angle X-ray scattering by nanomaterials. In: Klein L, Aparicio M, Jitianu A, editors. Handbook of Sol-Gel Science and Technology. Switzerland: Springer International Publishing; 2018. p. 1185-1230.

5. Fratzl P. Small-angle scattering in materials science - a short review of applications in alloys, ceramics and composite materials. Journal of Applied Crystallography. 2003;36(3):397-404.

6. Lamas DG, Oliveira Neto M, Kellermann G, Craievich AF. X-ray diffraction and scattering of nanomaterials. In: Roz AL, Leite FL, Ferreira M, Oliveira ON, editors. Nano Characterization Techniques. Oxford: Elsevier; 2017. p. 152-178. 
7. Zhu D, Porter AL. Automated extraction and visualization of information for technological intelligence and forecasting. Technological Forecasting and Social Change. 2002;69:495-506.

8. Craievich AF. The International Conference on Small-Angle Scattering - Past and Future (Foreword). Journal of Applied Crystallography. 2003;36(3-1):1-3.

9. Craievich AF, Fischer H. Quantitative analysis and relevant features of the scientific literature related to SAXS and SANS. Journal of Physics: Conference Series. 2010;247(1):012003.

10. Gutberlet T, Tunger D, Zeitler P, Bruckel T. Do neutrons publish? A neutrons publication survey 2005-2015. Neutron News. 2018;29(2):18-24.

11. Dabbousi BO, Rodriguez-Viejo J, Mikulec FV, Hene JR, Mattousi H, Ober R, et al. (CSe)ZnS core-shell quantum dots. Synthesis and characterization of a size series of highly luminescent nanocrystallites. Journal of Physical Chemistry: B. 1997;101(46):9463-9475.

12. Terech P, Weiss RG. Low molecular mass gelators of organic liquids and the properties of their gels. Chemical Reviews. 1997;97(8):3133-3159.

13. Decher G, Hong JD, Schmitt J. Buildup of ultrathin multilayer films by a self-assembly process consecutively alternating adsorption of anionic and cationic polyelectrolytes on charged surfaces. Thin Solid Films. 1992;210-211(Pt 2):831-835.

14. Svergun DI. Determination of the regularization parameter in indirect-transform methods using perceptual criteria. Journal of Applied Crystallography. 1992;25(4):495-503.

15. Svergun D, Barberato C, Koch MHJ. CRYSOL - a program to evaluate X-ray solution scattering of biological macromolecules from atomic coordinates. Journal of Applied Crystallography. 1995;28(6):768-773.
16. Konarev PV, Volkov VV, Sokolova AV, Koch MHJ, Svergun DI. PRIMUS: a Windows PC-based system for small-angle scattering data analysis. Journal of Applied Crystallography. 2003;36:1277-1282.

17. Hostetler MJ, Wingate JE, Zhong CJ, Harris JE, Vachet RW, Clark MR, et al. Alkanethiolate gold cluster molecules with core diameters from 1.5 to $5.2 \mathrm{~nm}$ : core and monolayer properties as a function of core size. Langmuir. 1998;14(1):17-30.

18. Estroff LA, Hamilton AD. Water gelation by small organic molecules. Chemical Reviews. 2004;104(3):1201-1218.

19. Fetters LJ, Lohse DJ, Richter D, Witten TA, Zirkel A. Connection between polymer molecular-weight, density, chain dimensions, and melt viscoelastic properties. Macromolecules. 1994;27(17):4639-4647.

20. Svergun DI. Restoring low-resolution structure of biological macromolecules from solution scattering using simulated annealing. Biophysical Journal. 1999;76(6):2879-2886.

21. Volkov VV, Svergun DI. Uniqueness of ab initio shape determination in small-angle scattering. Journal of Applied Crystallography. 2003;36(3):860-864

22. Zong XH, Kim K, Fang D, Ran S, Hsiao BS, Chu B. Structure and process relationship of electrospun bioabsorbable nanofiber membranes. Polymer. 2002;43(16):4403-12.

23. Shimizu T, Matsuda M, Minamikaya H. Supramolecular nanotube architectures based on amphiphilic molecules. Chemical Reviews. 2005;105(4):1401-1443.

24. Glatter O. A new method for evaluation of small-angle scattering data. Journal of Applied Crystallography. 1977;10(5):415-421.

25. Ipohorski M, Bonfiglioli A. Pre-precipitation in AlZn and AlZn alloys studied by small-angle X-ray scattering. Journal of Materials Science. 1967;2:371-7.

26. Craievich AF. Small-angle scattering study of phase separation in a $\mathrm{B}_{2} \mathrm{O}_{3}-\mathrm{PbO}-\mathrm{Al}_{2} \mathrm{O}_{3}$ glass. Physics and Chemistry of Glasses. $1975 ; 16: 133-8$. 